\title{
Nicotine Increases Codeine Analgesia Through the Induction of Brain CYP2D and Central Activation of Codeine to Morphine
}

\author{
Douglas M McMillan' and Rachel F Tyndale*,I \\ 'Campbell Family Mental Health Research Institute, Centre for Addiction and Mental Health (CAMH) and Departments of Psychiatry, \\ Pharmacology and Toxicology, University of Toronto, Toronto, Ontario, Canada
}

\begin{abstract}
CYP2D metabolically activates codeine to morphine, which is required for codeine analgesia. Permeability across the blood-brain barrier, and active efflux, suggests that initial morphine in the brain after codeine is due to brain CYP2D metabolism. Human CYP2D is higher in the brains, but not in the livers, of smokers and 7-day nicotine treatment induces rat brain, but not hepatic, CYP2D. The role of nicotineinduced rat brain CYP2D in the central metabolic activation of peripherally administered codeine and resulting analgesia was investigated. Rats received 7-day nicotine (I mg/kg subcutaneously) and/or a single propranolol (CYP2D mechanism-based inhibitor; $20 \mu g$ intracerebroventricularly) pretreatment, and then were tested for analgesia and drug levels following codeine (20 mg/kg intraperitoneally) or morphine $(3.5 \mathrm{mg} / \mathrm{kg}$ intraperitoneally), matched for peak analgesia. Nicotine increased codeine analgesia (I.59X AUC $0-30$ min vs vehicle; $p<0.00 \mathrm{I}$ ), while propranolol decreased analgesia (0.56X; $p<0.05)$; co-pretreatment was similar to vehicle controls $(\mathrm{I} .23 \mathrm{X} ; p>0.1)$. Nicotine increased, while propranolol decreased, brain, but not plasma, morphine levels, and analgesia correlated with brain $(p<0.02)$, but not plasma ( $p>0.4$ ), morphine levels after codeine. Pretreatments did not alter baseline or morphine analgesia. Here we show that brain CYP2D alters drug response despite the presence of substantial first-pass metabolism of codeine and further that nicotine induction of brain CYP2D increases codeine response in vivo. Thus variation in brain CYP2D activity, due to genetics or environment, may contribute to individual differences in response to centrally acting substrates. Exposure to nicotine may increase central drug metabolism, not detected peripherally, contributing to altered drug efficacy, onset time, and/or abuse liability.

Neuropsychopharmacology (2015) 40, I804-1812; doi:I0.1038/npp.2015.32; published online I8 February 2015
\end{abstract}

\section{INTRODUCTION}

The majority of cytochrome P450 (CYP)-mediated drug metabolism occurs in the liver; however, resulting plasma drug and/or metabolite levels do not always correlate with therapeutic effect (Ding and Kaminsky, 2003). Extra-hepatic CYP activity, specifically in the brain, may influence targettissue drug concentration and effect (Krishna and Klotz, 1994; Michels and Marzuk, 1993). Experimentally, this has been explored, for example, by inhibiting rat brain CYP2B, which decreased the inactivation of the sedative-hypnotic propofol, resulting in a prolonged propofol effect and decreased the activation of the organophosphate chlorpyrifos reducing neurotoxicity (Khokhar and Tyndale, 2011, 2012).

CYP2D6 (herein referred to as CYP2D for all species) metabolizes a large proportion of drugs acting in the central nervous system (CNS) (eg, amphetamine, haloperidol, and

* Correspondence: Dr RF Tyndale, Departments of Psychiatry, Pharmacology and Toxicology, University of Toronto, Medical Sciences Building Room 4326, I King's College Circle, Toronto, Ontario M5S IA8, Canada, Tel: 416978 6374, Fax: 4169786395 ,

E-mail: r.tyndale@utoronto.ca

Received 17 October 2014; revised 21 January 2015; accepted 26 January 2015; accepted article preview online 29 January 2015 antidepressants), as well as endogenous neurochemicals (Zanger et al, 2004). The human CYP2D gene is genetically polymorphic, resulting in a wide range of metabolism phenotypes (Gaedigk et al, 2008), while brain CYP2D levels are altered by common compounds and increase with age (Mann et al, 2012; Miksys and Tyndale, 2004; Warner and Gustafsson, 1994; Yue et al, 2008). Rat and monkey brain, but not liver, CYP2D is induced by nicotine pretreatment, consistent with higher levels of human brain, but not liver, CYP2D in smokers (Miksys and Tyndale, 2004; Miller et al, 2014; Yue et al, 2008). Thus the combination of genetics, environmental inducers, and age contribute to a wide range of brain CYP2D activity, which may alter the metabolism and efficacy of CNS-acting substrate drugs.

CYP2D metabolizes codeine to its primary analgesic metabolite morphine, a step required for codeine analgesia (Adler et al, 1955; Chen et al, 1991; Pert and Snyder, 1973; Sindrup et al, 1992; Sindrup et al, 1990). This occurs in the liver, after which morphine crosses the blood-brain barrier (BBB) to interact with opioid receptors in the CNS and elicit analgesia. However, morphine is less permeable across the BBB than codeine and is actively effluxed (Bouw et al, 2000; Oldendorf et al, 1972). Coupled with this, brain concentrations of morphine after codeine administration do not 
appear to be solely attributable to transfer of morphine across the BBB (Chen et al, 1990; Zhou et al, 2013). Thus, following codeine administration, the initial morphine present in the brain, and initial analgesia, may be due to brain CYP2D-mediated metabolism, rather than hepatic metabolism.

Our objectives were to model the role of human brain CYP2D in response to centrally acting drugs, through investigating rat brain CYP2D metabolic activation of codeine to morphine, in the presence of hepatic first-pass metabolism, in a rat model of codeine analgesia. Rat brain CYP2D has the ability to metabolize human CYP2D probe drugs and can be inhibited and induced in vivo, independently of hepatic CYP2D, allowing the modelling of a wide range of CYP2D activity within the brain, as postulated to exist in a heterogeneous population (Hiroi et al, 2002; Zhou et al, 2013). Whether the nicotine induction of brain CYP2D is sufficient to alter drug response in vivo, using a peripheral route of drug administration with substantial first-pass metabolism by the liver, is unknown. This study aims to demonstrate that variable brain CYP2D alters substrate drug metabolism and efficacy and provides further understanding to the functional role of CYP enzymes in the brain.

\section{MATERIALS AND METHODS}

\section{Animals}

Adult male Wistar rats (250-500 g; Charles River, St-Constant, QC, Canada) were housed in pairs or triplets with ad libitum access to food and water. Rats were maintained under a 12-h artificial light/dark cycle with experimentation occurring during the light cycle. Stress was reduced through acclimation to experimenters and apparatuses. All procedures were approved by the Animal Care Committee at the University of Toronto.

\section{Drug Treatment}

Codeine phosphate and morphine sulfate (PCCA, London, Canada) were dissolved in saline $(0.9 \% \mathrm{NaCl} ; \mathrm{pH} 7)$ and distilled water, respectively, and injected intraperitoneally (i.p.). The doses of codeine $(20 \mathrm{mg} / \mathrm{kg})$ and morphine $(3.5 \mathrm{mg} / \mathrm{kg})$ were chosen to match peak analgesia after testing multiple doses of codeine $(20,25$ and $30 \mathrm{mg} / \mathrm{kg})$ and morphine $(0.5,1,2$, and $3.5 \mathrm{mg} / \mathrm{kg})$. Propranolol hydrochloride (Sigma-Aldrich, Oakville, Canada) was dissolved in artificial cerebrospinal fluid to deliver $20 \mu \mathrm{g}$ base in $4 \mu \mathrm{l}$ via intracerebroventricular (i.c.v.) injection $24 \mathrm{~h}$ prior to codeine or morphine administration. Propranolol is a CYP2D mechanism-based inhibitor, metabolized by CYP2D; the metabolite covalently binds and inactivates CYP2D (Narimatsu et al, 2001). Propafenone hydrochloride (Sigma-Aldrich), a CYP2D competitive inhibitor (Xu et al, 1995), was dissolved in a $20 \%(\mathrm{w} / \mathrm{v})$ solution of 2 -hydroxypropyl- $\beta$-cyclodextrin (Sigma-Aldrich) in water to deliver $40 \mu \mathrm{g}$ base in $4 \mu \mathrm{l}$ i.c.v., 5 min prior to codeine or morphine administration. Two mechanistically and structurally distinct CYP2D inhibitors were used to reduce the possibility of off-target effects other than inhibition of codeine metabolism. Nicotine bitartrate (Sigma-Aldrich) was dissolved in saline and given subcutaneously (s.c.) at $1 \mathrm{mg}$ base/kg daily for 7 days, which results in elevated rat brain, but not liver, CYP2D levels as previously described (Yue et al, 2008).

\section{Cannulation Surgeries for Multiple i.c.v. Injections}

Rats utilized for brain CYP2D-inhibition experiments were surgically implanted with i.c.v. cannulas into their right lateral ventricle (Zhou et al, 2013). Screw anchors (BASi, West Lafayette, USA) were implanted into the skull to serve as an anchor to the DuraLay inlay pattern resin (Reliance Dental Mfg. Co., Worth, USA) used as an adhesive for the implanted cannula.

\section{Nociceptive Testing of Codeine and Morphine Analgesia}

Analgesia was measured by the tail-flick reflex test through the use of a tail-flick meter (Columbus Instruments, Columbus, OH, USA) (D'Amour and Smith, 1941). A thermal stimulus is applied to the rat's tail, $-5 \mathrm{~cm}$ from the distal end, and the time of onset of stimulus to a vigorous tail flick is recorded as the tail-flick latency (TFL). Prolongation of the TFL is an indication of both spinally and supra-spinally mediated analgesia (Le Bars et al, 2001). The thermal strength of the tail-flick meter was adjusted to obtain baseline TFLs around $3 \mathrm{~s}$; a cutoff of $10 \mathrm{~s}$ was used to avoid damage. TFLs were measured twice in each rat $24 \mathrm{~h}$ prior to experimentation, and the mean was used as the individual rat's baseline TFL. Analgesia was expressed as a percentage of the maximal possible effect (\%MPE) (Dewey and Harris, 1971).

$\% \mathrm{MPE}=\frac{(\text { postinjection latency }- \text { baseline latency })}{(\text { maximum cutoff }- \text { baseline latency })} \times 100 \%$

\section{Analgesia Experimental Design}

For pretreatment experiments, codeine or morphine was given $24 \mathrm{~h}$ after i.c.v. pretreatment injection (propranolol or vehicle) and/or $8 \mathrm{~h}$ after the last s.c. pretreatment injection (nicotine or saline). After opioid administration, analgesia was assessed by TFL every $5 \mathrm{~min}$ for $30 \mathrm{~min}$ and then every $10 \mathrm{~min}$ for the next $30 \mathrm{~min}$. Within-animal designs were used where possible in which, after a 1-week washout period, rats were crossed over to a comparator pretreatment group and retested or killed for pharmacokinetic analysis.

\section{Plasma and Brain Sample Preparation}

For analysis of codeine and morphine from the plasma and brain, rats were pretreated, then administered codeine, tested for TFL at 15 min postinjection, and killed by decapitation. Trunk blood was centrifuged at $5000 \mathrm{~g}$ for $10 \mathrm{~min}$. Halfbrains were homogenized in $1: 3(\mathrm{w} / \mathrm{v}) \quad 0.01 \mathrm{~N} \mathrm{HCl}$ and centrifuged at $5000 \mathrm{~g}$ for $10 \mathrm{~min}$. Nicotine plasma samples were obtained on the seventh day of nicotine pretreatment via saphenous-vein blood draws.

\section{HPLC Measurement of Plasma and Brain Drug/Metabolite Levels}

Plasma and brain homogenate samples were analyzed as previously described (Zhou et al, 2013). In brief, $100 \mu$ of 
solid phase extract was analyzed by HPLC with ultraviolet detection at $214 \mathrm{~nm}$ (Agilent 1200 Separation Module, Palo Alto, CA, USA). Morphine, codeine, and internal standard (1 $\mu \mathrm{g}$ 2-benzoxazolinone) were separated on an Agilent ZORBAX SB-C18 Column $(250 \mathrm{~mm} \times 4.6 \mathrm{~mm}$ I.D.; particle size, $5 \mu \mathrm{m}$ ), with the retention times of 4.1, 9.0 and $16.7 \mathrm{~min}$, respectively. The mobile phase used was methanol: phosphate buffer $(29.3: 70.7(\mathrm{v} / \mathrm{v}) ; \mathrm{pH} 5.8)$ with a flow rate of $1 \mathrm{ml} / \mathrm{min}$. The limits of quantification were 5 and $10 \mathrm{ng} / \mathrm{ml}$ ( 5 and $50 \mathrm{ng} / \mathrm{g}$ for brain tissue) for morphine and codeine, with an extraction efficiency of 76.9 and $90.1 \%$, respectively. Nicotine and cotinine were quantified by HPLC (Siu et al, 2006).

\section{Statistical Analyses}

Data were analyzed through one-way or two-way repeatedmeasures ANOVA with Bonferroni corrections or unpaired (between animal) and paired (within animal) one-tailed $t$-tests, as indicated.

\section{RESULTS}

\section{Analgesic Profiles and Morphine Concentrations Suggest Involvement of Brain CYP2D in Codeine Metabolism and Initial Codeine Analgesia}

Codeine $(20 \mathrm{mg} / \mathrm{kg})$ resulted in a peak of analgesia around 15 min ( $n=22$; Figure 1a) with morphine metabolite levels detectable in both plasma and brain $(n=5$; Figure $1 \mathrm{~b}$ and c). This codeine dose used throughout provided analgesia below maximal \%MPE, allowing for measurement of both increases and decreases in analgesia. Morphine $(3.5 \mathrm{mg} / \mathrm{kg})$ matched the peak analgesia level obtained from codeine, however, later at $30 \mathrm{~min}(n=8$; Figure 1a). At $15 \mathrm{~min}$, the morphine dose produced similar plasma morphine levels to those from codeine $(p>0.3 ; n=10$; Figure $1 \mathrm{~b})$, but lower brain morphine levels $(p<0.02$; Figure 1c), consistent with lower analgesia at this time point. This suggests that the early analgesia peak at $15 \mathrm{~min}$ following codeine, along with the high levels of brain morphine present, was due to morphine formed by central (brain) metabolism. The delayed peak in analgesia after morphine suggests that peripheral morphine, as compared with the more lipophilic codeine, crosses the BBB more slowly, therefore eliciting analgesia at a later time.

\section{Pretreatment with Two Distinct CYP2D Inhibitors (i.c.v.) Decreased the First 30 min of Codeine-Induced Analgesia but Did Not Affect Morphine-Induced Analgesia}

With a focus on the time frame (0-30 min postinjection) hypothesized to be associated with brain metabolism of codeine to morphine and the resulting peak in codeineinduced analgesia, the effect of inhibiting brain CYP2D was assessed using two structurally and mechanistically distinct CYP2D inhibitors. Compared with vehicle pretreatment, $20 \mu \mathrm{g}$ propranolol (mechanism-based inhibitor) pretreatment resulted in significantly lower \%MPE at numerous time points $(n=16 /$ group; Figure $2 \mathrm{a})$ after codeine $(20 \mathrm{mg} / \mathrm{kg})$ administration, producing a significant reduction of the analgesic $\mathrm{AUC}_{0-30 \mathrm{~min}}(p<0.01$; Figure $2 \mathrm{~b})$. Likewise, $40 \mu \mathrm{g}$
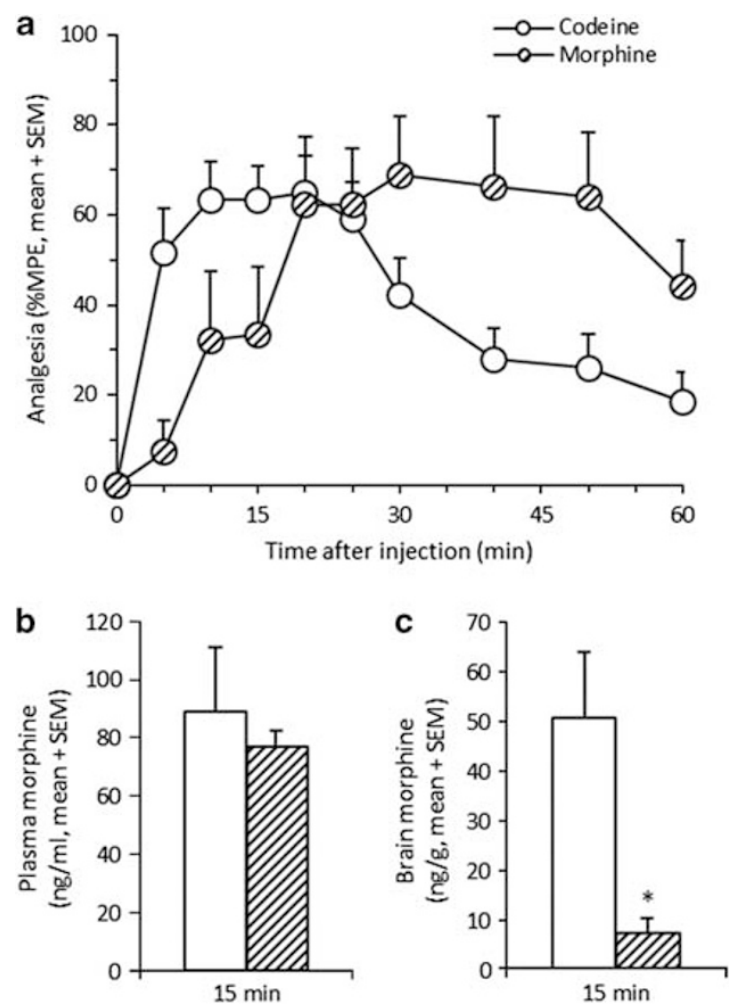

Figure I The analgesic time course following codeine and morphine suggests peripheral morphine levels do not predict central morphine levels or analgesia. Codeine $(20 \mathrm{mg} / \mathrm{kg})$ produced an analgesic peak around $15 \mathrm{~min}$ postinjection $\left(T_{\text {MAX }}=13+1.8\right.$ min, mean $\left.+\mathrm{SEM} ; n=22 ; a\right)$. A morphine dose $(3.5 \mathrm{mg} / \mathrm{kg})$, chosen to match peak analgesia from codeine, resulted in an equivalent albeit later analgesia peak around $30 \mathrm{~min}$ postinjection $\left(T_{\text {MAX }}=27+4.9 \mathrm{~min} ; n=8\right)$. At $15 \mathrm{~min}$ postinjection, the time of the codeine analgesia peak, the morphine dose $(n=10)$ produced similar plasma morphine levels to the codeine dose $(n=5 ; b)$, and lower brain morphine levels (c) consistent with the lower analgesia. A between-animal study design was used; *p $<0.05$ compared with codeine $(20 \mathrm{mg} / \mathrm{kg})$ using an unpaired $t$-test.

propafenone (competitive inhibitor) pretreatment resulted in significantly lower \%MPE ( $n=6$ /group; Figure $2 \mathrm{~d})$, and analgesic $\mathrm{AUC}_{0-30 \text { min }}$ after codeine administration $(p<0.05$; Figure 2e).

As morphine is the active analgesic metabolite of codeine and not further metabolized by CYP2D, its analgesia should not be altered by changes in CYP2D activity. Compared with vehicle pretreatment, neither propranolol nor propafenone pretreatment altered \%MPE ( $p>0.1$ at all time points) or analgesic AUC $(p>0.1)$ across any time frame after morphine administration ( $n=5$ /group; illustrated for $\mathrm{AUC}_{0-30 \mathrm{~min}}$ and $\mathrm{AUC}_{30-60 \text { min }}$ in Figure $2 \mathrm{c}$ and $\mathrm{f}$ ).

Pretreatment with CYP2D inhibitors also had no effect on baseline nociception; there was no difference between TFL prior to $(3.52+0.21$, mean + SEM $)$ and $24 \mathrm{~h}$ after vehicle $(3.64+0.16 \mathrm{~s})$ or $(3.72+0.20 \mathrm{~s})$ propranolol pretreatment $(n=16 ; p>0.2)$, or TFL prior to $(3.03+0.14 \mathrm{~s})$ and $5 \mathrm{~min}$ after vehicle $(3.13+0.14 \mathrm{~s})$ or $(3.18+0.16 \mathrm{~s})$ propafenone pretreatment $(n=6 ; p>0.2)$. Together, this indicated that CYP2D inhibitor pretreatments had no effect on analgesia that was unrelated to their effects on codeine metabolism. 

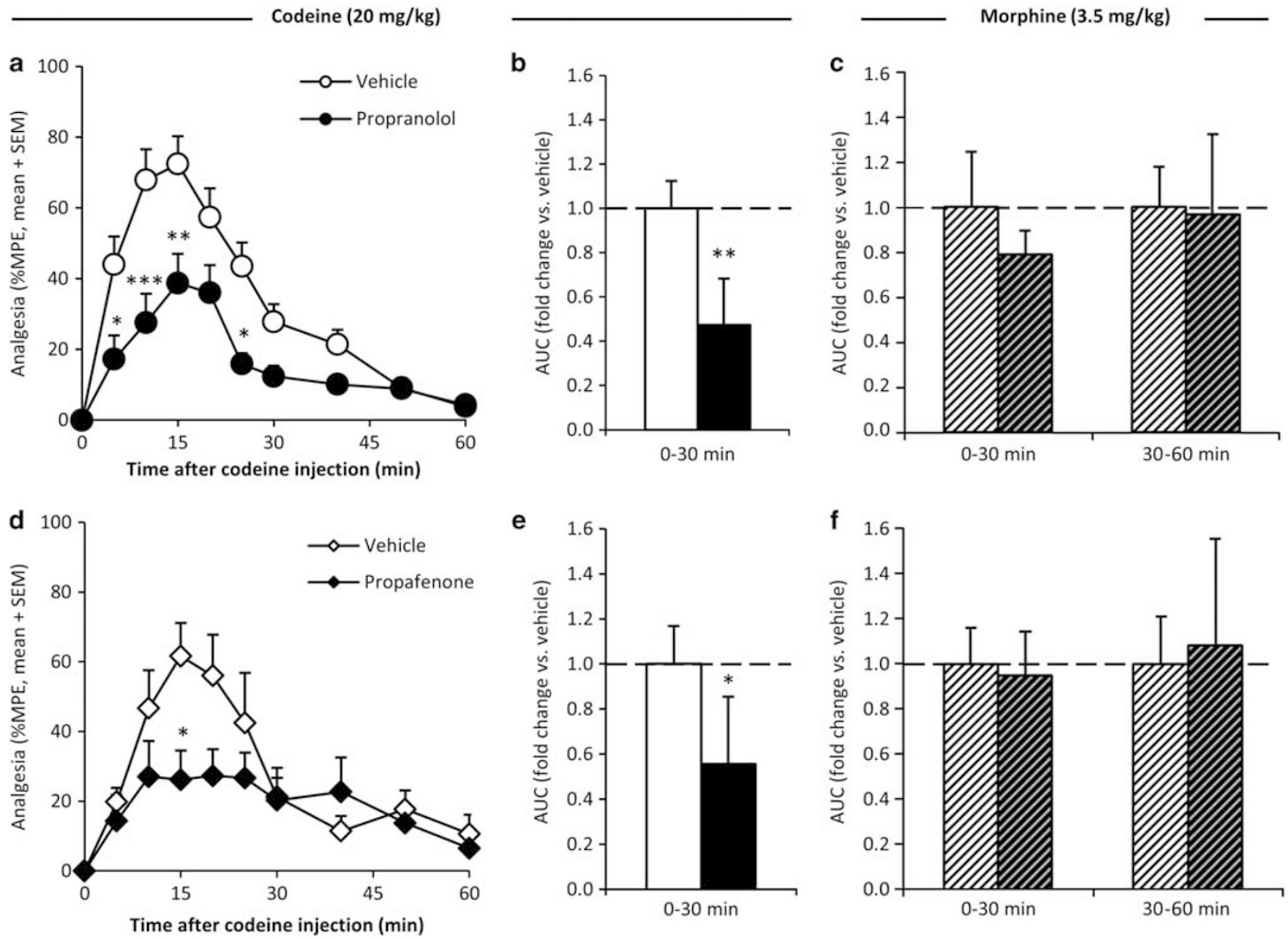

Figure 2 Inhibiting brain CYP2D reduced the first 30 min of codeine-induced analgesia but had no effect on morphine-induced analgesia. Compared with vehicle pretreatment, propranolol pretreatment (darker bar) resulted in significantly lower analgesia (\%MPE; a) and area under the analgesia-time curve (AUC; b) for 0-30 min after codeine administration ( $n=16 /$ group). Propranolol pretreatment (darker bars) did not result in a difference in analgesic AUC $\mathrm{C}_{-30}$ min or

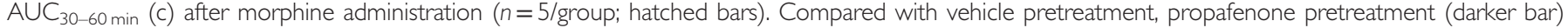

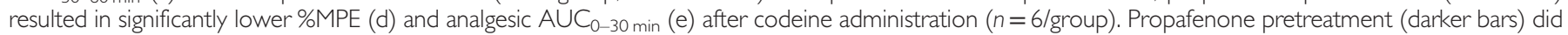

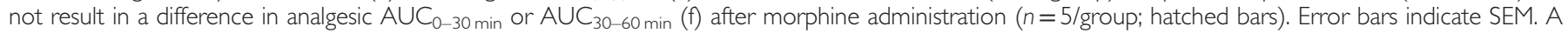
within-animal study design was used; for analgesia-time curves, $* p<0.05$, $* * p<0.01$, and $* * * *<0.00$ I using repeated-measures ANOVA with Bonferroni post hoc. For AUCs, ${ }^{*} p<0.05$, ** $p<0.0$ I using paired t-tests.

\section{7-Day Pretreatment with Nicotine, to Induce Brain CYP2D, Increased the First 30 min of Codeine-Induced Analgesia but Did Not Affect Morphine-Induced Analgesia}

Nicotine administration induces rat brain CYP2D without altering hepatic CYP2D; rats were administered nicotine $(1 \mathrm{mg} / \mathrm{kg}$; s.c.) or vehicle (saline, $1 \mathrm{ml} / \mathrm{kg}$; s.c.) and tested at $8 \mathrm{~h}$ after the seventh and final injection (Figure 3a), when brain CYP2D is significantly induced (Yue et al, 2008). On the final day of nicotine pretreatment, plasma nicotine levels peaked within the first $30 \mathrm{~min}$ and were undetectable at $8 \mathrm{~h}$ postinjection, the time of codeine or morphine administration and analgesia testing $(n=16$; Figure $3 \mathrm{~b})$. Compared with vehicle pretreatment, 7 days of nicotine pretreatment resulted in significantly higher \%MPE ( $n=20$ /group; Figure $3 \mathrm{a}$ ) after codeine administration, producing a significantly higher $\mathrm{AUC}_{0-30 \text { min }}(p<0.03$; Figure $3 \mathrm{~b})$.

The effect of nicotine pretreatment on morphine analgesia $(3.5 \mathrm{mg} / \mathrm{kg})$ was also tested; if nicotine's actions on increasing codeine-induced analgesia were via induced brain
CYP2D, no change in morphine-induced analgesia would be expected. Compared with vehicle pretreatment, nicotine pretreatment did not result in significantly altered \%MPE $(p>0.3$ at all time points) or analgesic AUC ( $p>0.3)$ across any time frame after morphine administration $(n=16 /$ group; illustrated for $\mathrm{AUC}_{0-30 \text { min }}$ and $\mathrm{AUC}_{30-60 \text { min }}$ in Figure 3e).

The effect of 7-day nicotine pretreatment on codeine and morphine analgesia from the tail-flick assay was also tested using the hot-plate assay (Eddy and Leimbach, 1952; Rowland et al, 1994). Compared with vehicle, nicotine pretreatment resulted in significantly higher \%MPE (Supplementary Figure S1a) and a 2.46 -fold greater $\mathrm{AUC}_{0-30 \mathrm{~min}}(p<0.01$; $n=8$ /group; Supplementary Figure S1b) after codeine administration, with no change in \%MPE ( $p>0.2$ at all time points) or AUC ( $p>0.3)$ across any time frame after morphine administration ( $n=5$ /group; Supplementary Figure S1c in the Supplementary Materials S1).

TFLs were assessed prior to daily nicotine injections and at $8 \mathrm{~h}$ postinjection (the time at which codeine and morphine were tested). There was no change in preinjection TFL (Day 
a

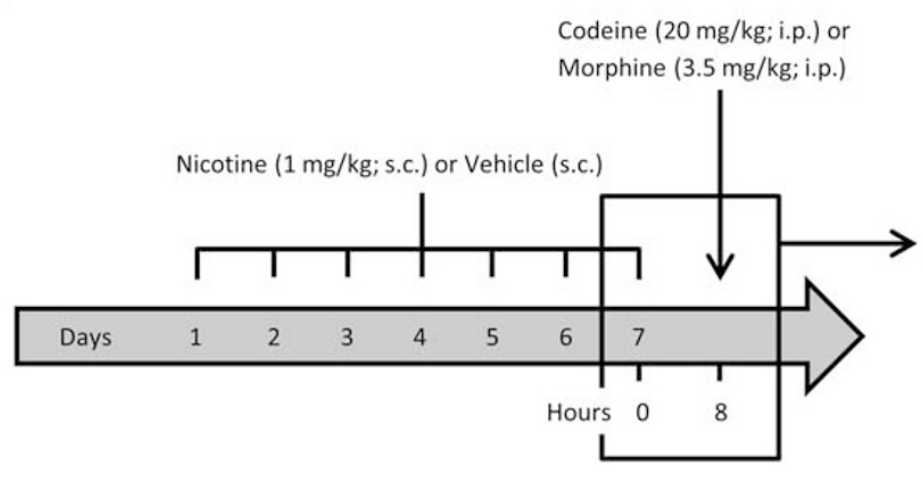

Codeine $(20 \mathrm{mg} / \mathrm{kg})$

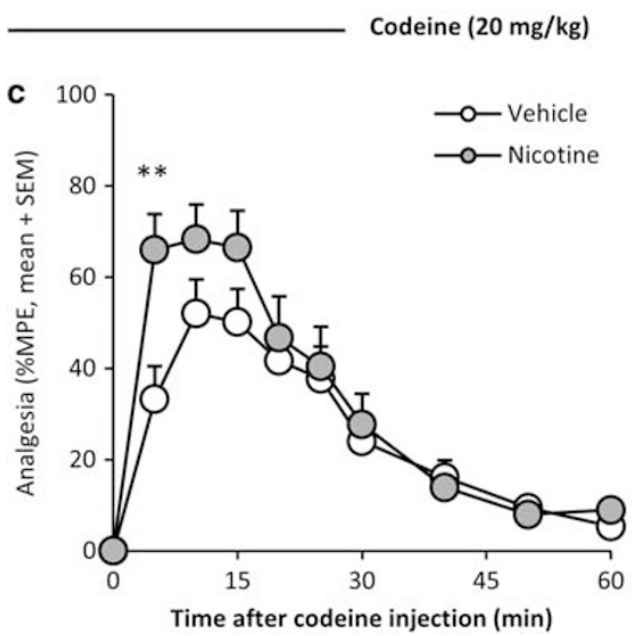

b

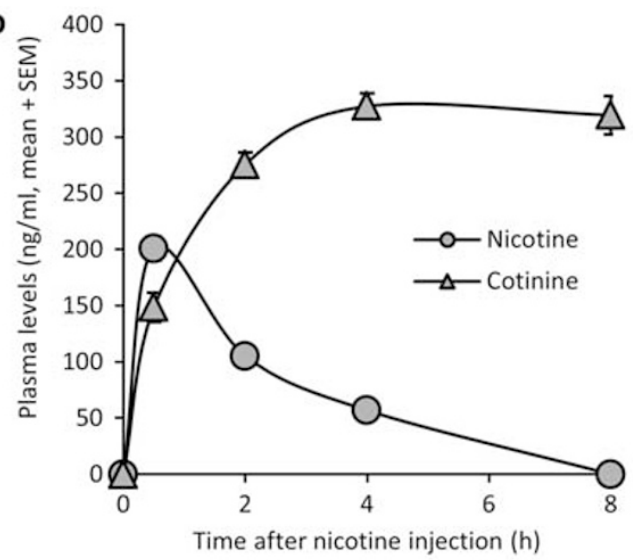

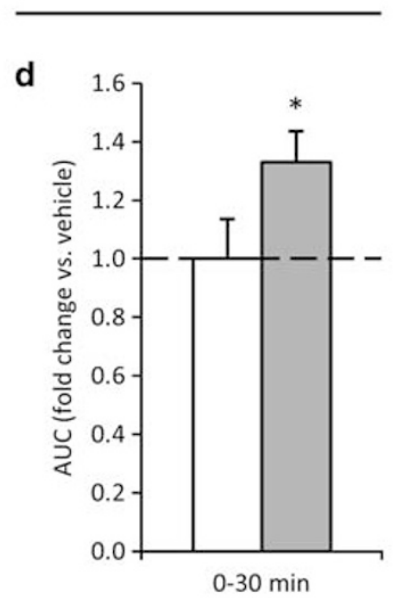

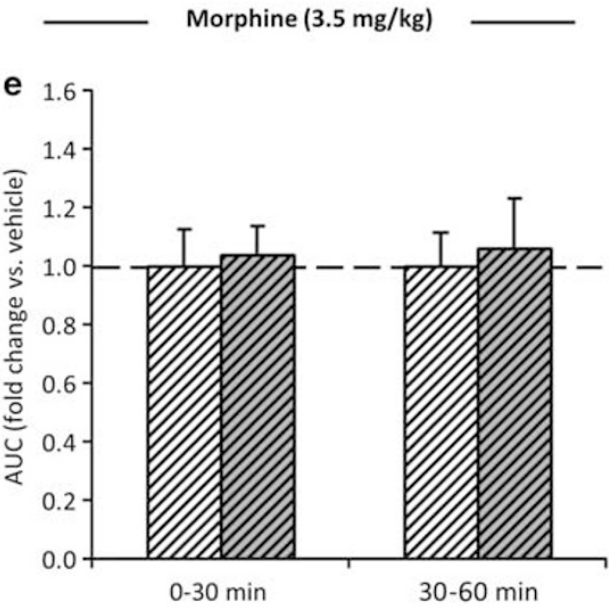

Figure 3 Nicotine-mediated induction of brain CYP2D increased codeine analgesia and had no effect on morphine-induced analgesia. Rats were administered nicotine (I mg/kg; s.c.) or saline vehicle (s.c.) once daily for 7 days as outlined (a). As measured on the final day of nicotine pretreatment, plasma nicotine levels peaked within the first 30 min and were undetectable by $8 \mathrm{~h}$, the time of codeine or morphine administration ( $n=16$; b). Mean plasma nicotine levels averaged over $8 \mathrm{~h}$ was approximately $70 \mathrm{ng} / \mathrm{ml}$ and over $24 \mathrm{~h}$ was approximately $25 \mathrm{ng} / \mathrm{ml}$. Compared with vehicle pretreatment, nicotine pretreatment (darker bar) resulted in significantly higher \%MPE (c) and analgesic $A \cup C_{0-30 ~ m i n}$ (d) after codeine administration ( $\left.n=20 / g r o u p\right)$. Seven days of nicotine

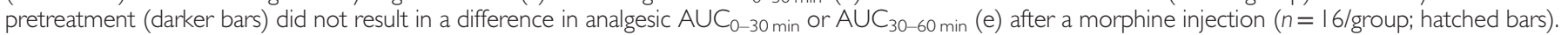
Error bars indicate SEM. A within-animal study design was used; for analgesia-time curves, *** $<0.01$ using repeated-measures ANOVA with Bonferroni post hoc. For AUCs, ${ }^{*} p<0.05$ using paired t-tests.

1: $5.4+0.5 \mathrm{~s}$, mean + SEM; Day 7: $5.3+0.4 \mathrm{~s} ; p=0.39)$ or $8 \mathrm{~h}$ postinjection TFL (Day 1: $5.1+0.5 \mathrm{~s}$; Day 7: $4.7+0.3 \mathrm{~s}$; $p=0.20)$ and no change in TFL between preinjection and $8 \mathrm{~h}$ postinjection $(p>0.1)$ across each of the 7 days of nicotine injections. Together, with the lack of effect on morphine analgesia, this suggests that nicotine (as tested here) did not alter nociception during codeine and morphine testing, suggesting, instead, a direct effect on codeine analgesia via induction of brain CYP2D.

\section{Pretreatment with a Mechanism-Based Brain CYP2D Inhibitor Blocked the Effect of Nicotine Induction of Brain CYP2D on Codeine Analgesia}

Compared with vehicle pretreatment, when animals were pretreated with 7 days of nicotine, there was a significant increase in \%MPE ( $n=12$ /group; Figure $4 \mathrm{a})$ and resulting increase in analgesic $\mathrm{AUC}_{0-30 \mathrm{~min}}$ after codeine $(p<0.001$; Figure $4 \mathrm{~b}$ ). Conversely, when animals were pretreated with propranolol, there was a significant decrease in \%MPE and analgesic $\mathrm{AUC}_{0-30 \text { min }}$ after codeine administration $(p<0.05)$.
Co-pretreatment of propranolol blocked the nicotine-induced increase in codeine analgesia, likely through inhibiting the induced levels of brain CYP2D, and resulted in similar codeine to the vehicle pretreatment $(p=0.8)$. There was no significant difference in baseline TFL between the four pretreatment groups $(p>0.3)$. Thus the effect of propranolol and nicotine pretreatment together resulted in no net change in codeine-induced analgesia, suggesting that they were acting in opposing directions via the same mechanism, presumably through modification of brain CYP2D activity.

\section{Variation in Brain CYP2D Altered Brain Metabolism of Codeine Without Affecting Hepatic CYP2D}

To investigate whether the alterations in codeine analgesia correlated with changes in codeine metabolism in vivo, brain and plasma codeine and morphine levels were assessed after administration of the combination of pretreatments used previously (Figure 4). There was a significant correlation between plasma and brain codeine levels $(r=0.60 ; p<0.001$; Figure 5a) but not between morphine levels $(r=0.14$; 

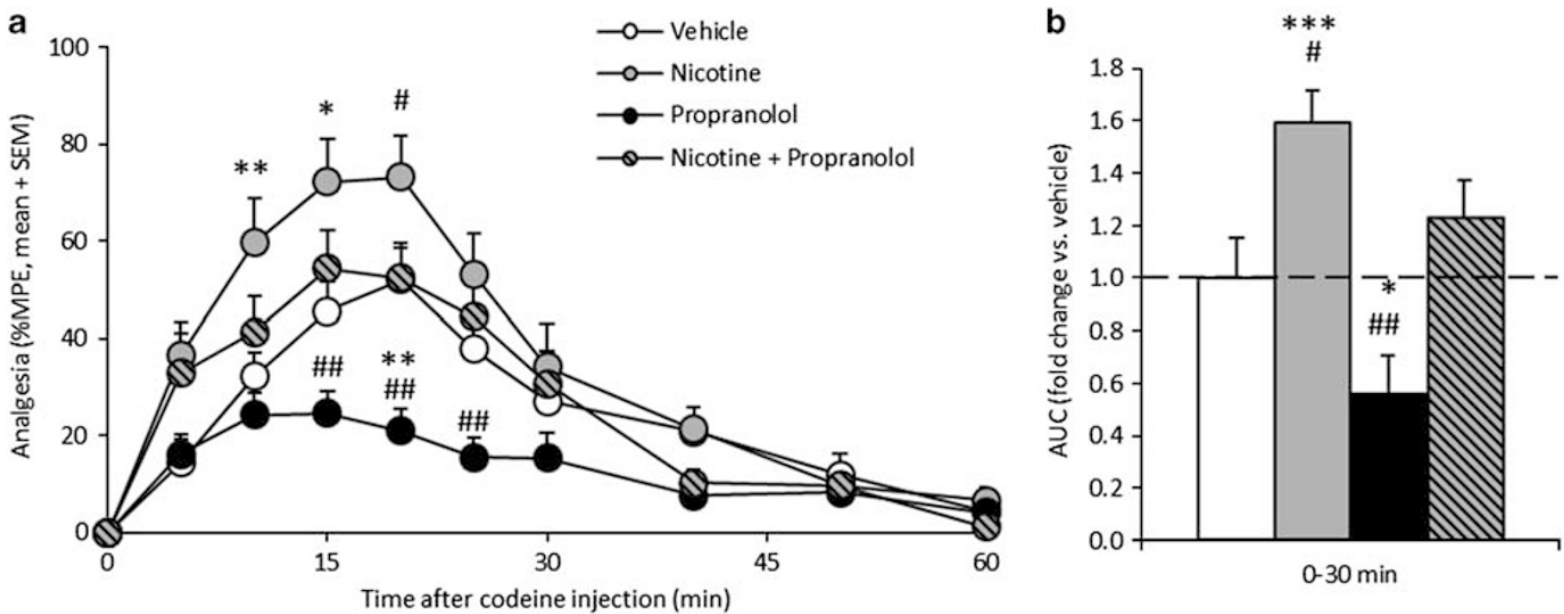

Figure 4 Nicotine induction of brain CYP2D increased the first 30 min of codeine-induced analgesia, an effect that was blocked by inhibiting brain CYP2D. Compared with vehicle pretreatment, 7 days of nicotine pretreatment or propranolol pretreatment alone resulted in significantly higher and lower,

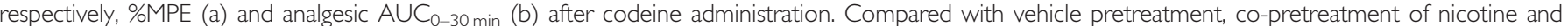
propranolol together resulted in a significantly different \%MPE and $\mathrm{UUC}_{0-30 \text { min }}$ than nicotine or propranolol pretreatment alone and no change in \%MPE or $A \cup C_{0-30 \text { min }}$ from vehicle pretreatment ( $n=12 /$ group). Error bars indicate SEM. A within-animal study design was used; $* p<0.05$, *** $p<0.0$ I, **** $p<0.00$ I, compared with vehicle, and ${ }^{\#} p<0.05,{ }^{\#} p<0.01$, compared with the combination pretreatment, using repeated-measures ANOVA with a Bonferroni post hoc test.

$p=0.26$; Figure $5 \mathrm{~b})$ at $15 \mathrm{~min}$ after codeine administration $(n=26)$. This suggests that at $15 \mathrm{~min}$ after codeine administration plasma morphine levels did not predict brain morphine levels, indicating the presence of barriers to morphine permeability across the $\mathrm{BBB}$. In the same analysis, analgesia at $15 \mathrm{~min}$ correlated with brain morphine levels $(r=0.41 ; p<0.02$; Figure $5 c)$ but not with plasma morphine $(r=0.01 ; p=0.48$; Figure $5 \mathrm{~d})$ at $15 \mathrm{~min}$ after codeine administration $(n=26)$, consistent with brain CYP2Dmediated codeine metabolism and resulting brain morphine levels, being responsible for codeine analgesia over the first 30 min after codeine administration.

Compared with vehicle pretreated rats $(48.5+2.0 \mathrm{ng} / \mathrm{g}$, mean + SEM), nicotine pretreated rats had significantly higher morphine levels in the brain $(54.7+2.4 \mathrm{ng} / \mathrm{g} ; p<0.02)$, propranolol pretreated trended towards lower morphine levels in the brain $(44.5+2.6 \mathrm{ng} / \mathrm{g} ; p=0.2)$, and rats co-pretreated with nicotine and propranolol had no difference in the brain morphine levels $(51.6+2.2 \mathrm{ng} / \mathrm{g} ; p=0.8)$ after codeine administration, all consistent with brain CYP2D-specific changes ( $n=8$ /group). The three active pretreatment paradigms did not alter plasma morphine levels $(p>0.7$, for all comparisons), suggesting that these pretreatments modified brain metabolism of codeine, without affecting hepatic metabolism.

\section{DISCUSSION}

This is the first study to demonstrate that brain CYP2D has a role in drug response even in the presence of substantial first-pass metabolism of codeine and further that nicotine induction of brain CYP2D can increase central drug metabolism and response in vivo. Previously, it was unknown whether the nicotine-induced levels of brain CYP2D were sufficient and possessed adequate cofactors in situ to alter CNS-acting drug metabolism and resulting drug effect in vivo. This is also the first study to show that
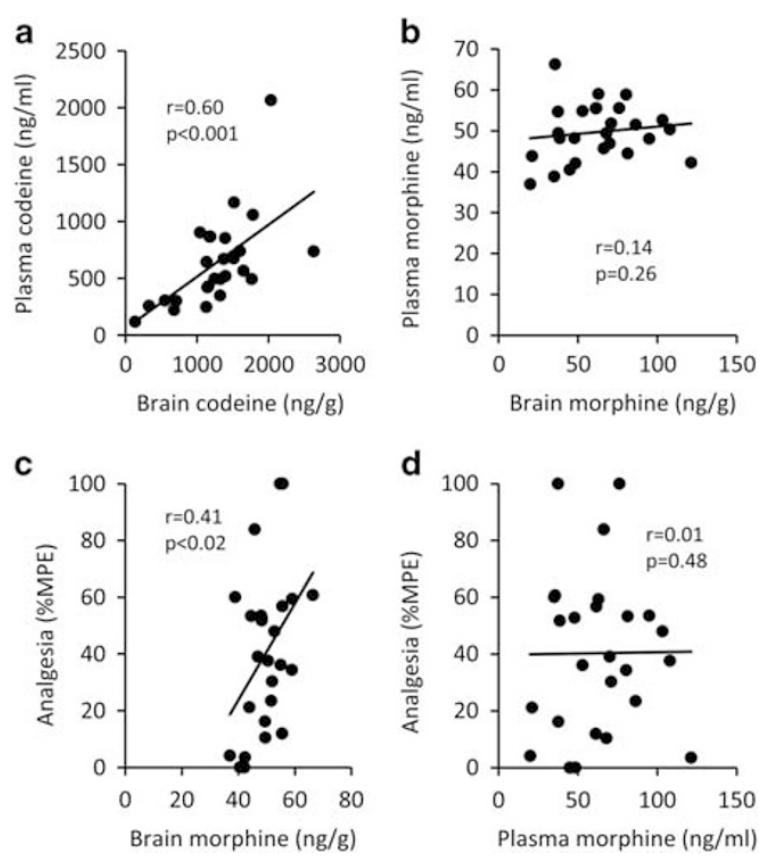

Figure 5 Codeine, but not morphine, levels correlate between plasma and brain; brain morphine levels reflect codeine analgesia. Plasma and brain codeine levels correlated significantly (a) at $15 \mathrm{~min}$, suggesting that codeine equilibrated quickly between the plasma and brain compartments. There was no correlation between plasma and brain morphine levels following codeine administration (b). Analgesia correlated with brain morphine levels (c) but not with plasma morphine (d) at 15 min after codeine administration $(n=26)$. Pearson correlations were used.

this nicotine induction of brain CYP2D alters drug response and can be blocked with brain CYP2D inhibitors, suggesting the importance of the interplay of compounds that alter brain activity on centrally acting drug effect, including for orally administered drugs. 
Codeine and morphine, given at doses resulting in equivalent plasma morphine levels at 15 min postinjection and peak analgesia, resulted in significantly different brain morphine levels and analgesia time lines, suggesting that hepatic metabolism of codeine to morphine was not solely responsible for the levels of brain morphine and centrally mediated analgesia after a codeine injection. Our findings suggest a significant role for variable brain metabolism in codeine analgesia; inhibiting brain CYP2D with two mechanistically distinct inhibitors decreased, while inducing brain CYP2D with nicotine increased, codeine analgesia and resulting brain morphine levels, effects that were selectively blocked by co-pretreatment. Furthermore, neither inhibiting nor inducing brain CYP2D altered baseline response, morphine, analgesia, or peripheral metabolism of codeine, suggesting that the pretreatments used to modify brain CYP2D did not have off-target effects on codeine's downstream mechanism of action.

The 7-day nicotine pretreatment paradigm resulted in average daily nicotine plasma levels of $25 \mathrm{ng} / \mathrm{ml}$ (Figure 3b), similar to those seen in human smokers $(20-50 \mathrm{ng} / \mathrm{ml})$ (Benowitz, 1999); similar to nicotine-treated animals, smoking is associated with higher human brain CYP2D (Mann et al, 2008; Miksys and Tyndale, 2004; Miller et al, 2014; Yue et al, 2008). Peripheral pharmacokinetics of codeine are similar in smokers and nonsmokers, consistent with our current animal studies, and with a lack of effect of smoking or nicotine treatment on hepatic CYP2D (Miller, 1990). Approximately $20 \%$ of the US population continues to smoke cigarettes; smokers have a greater risk of multi-drug use and dependency (Berg et al, 2013). Furthermore, the use of electronic cigarettes that deliver nicotine mimicking conventional cigarettes is increasing, particularly in adolescents (Dutra and Glantz, 2014). Opioid analgesics such as codeine, while widely accepted for the treatment of acute to chronic pain, are abused for their euphoric mood-altering properties, with the incidence of non-medicinal prescription opioid use increasing (Fischer et al, 2006). The reinforcing qualities of codeine come from its morphine metabolite, therefore for individuals with higher levels of brain CYP2D (eg, smokers) a more rapid exposure of the brain to morphine, through faster centrally mediated metabolic activation, could speed the onset time of codeine's reinforcing effects and increase its abuse liability (Kathiramalainathan et al, 2000; Tyndale et al, 1997). Increased central opiate activation may be one mechanism increasing the risk of smoking and opioid co-dependency (Richter and Ahluwalia, 2000). In support of this, the prevalence of repeated prescription frequency, and general non-medicinal prescription opioid use, is higher in both men and women with smoking dependence (Skurtveit et al, 2010). Although alternative mechanistic explanations exist, such as receptor cross-sensitization in the enhanced rewarding and psychomotor effects of morphine in rodents following nicotine treatment, nicotine's ability to alter drug metabolism within the brain may also contribute (Vihavainen et al, 2008).

In animals pretreated with chronic nicotine, there was a trend for a faster onset of analgesia; the $T_{\mathrm{MAX}}$ with brain CYP2D induction $(14.6+1.7 \mathrm{~min}$, mean + SEM) was shorter compared with vehicle pretreatment $(18.8+1.4 \mathrm{~min} ; p>0.1)$, brain CYP2D inhibition $(17.1+1.8 \mathrm{~min} ; p>0.4)$ and copretreatment $(17.1+1.8 \mathrm{~min} ; n=12$ /group; $p>0.5$ using a two-way ANOVA against brain CYP2D induction). A shift

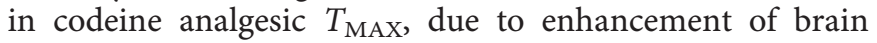
CYP2D metabolism, could contribute to a faster onset of codeine's clinical actions but may also increase its abuse liability. Individuals exposed to compounds that affect brain CYP2D levels and/or activity could present with varied profiles of both clinical effect and abuse potential to drugs that are activated (ie, codeine), or inactivated (ie, amphetamine) by CYP2D (Wu et al, 1997).

The absolute magnitude of the change in morphine levels, analgesia and $\mathrm{T}_{\text {MAX }}$ was likely blunted, due to the brain CYP2D inhibition paradigm employed, resulting from incomplete inhibition of CYP2D. A larger dose of propranolol ( $40 \mu \mathrm{g}$ base in $4 \mu \mathrm{l}$ ), which reduced analgesia further, inhibited hepatic CYP2D (determined by ex vivo metabolic assays) suggesting that propranolol had crossed the BBB into the periphery and obscured the ability to focus solely on brain metabolism (Zhou et al, 2013). An active search is underway for alternative molecular-inhibition options for future experiments. In addition, brain CYPs are highly localized and respond to inducers and inhibitors with complex patterns of regulation that are both tissue and cell-type specific (Yue et al, 2008). For this reason, the direct correlation between brain regions associated with antinociception and with CYP2D protein affected by induction and inhibition is unclear. Thus global brain inhibition and homogenates were used as a way of capturing a more general picture, however, likely resulted in a muting of the propranolol effect. Further studies into brain regions corresponding to high opioid receptor and CYP2D protein density are warranted to elucidate the importance of region-specific brain CYP2D alterations on codeine metabolism and analgesia.

Nicotine has an important role in modulating pain transmission. Activation of the $\alpha 4 \beta 2$ and $\alpha 7$ nicotinic acetylcholine receptor subtypes elicits analgesia and is purported to involve opioidergic neurons and endogenous opioid peptides; nicotine analgesia is decreased in $\mu$-opioid receptor knockout mice (Berrendero et al, 2002; Carstens et al, 2001; Damaj et al, 2000; Dhatt et al, 1995). Furthermore, nicotine dependence and withdrawal can affect both opioid- and noxious stimulus-induced analgesia involving these receptors in the nucleus accumbens (Schmidt et al, 2001). For these reasons, it was important to control for nicotine's own potential effects on nociception and opioid action. There were no detectable levels of nicotine at $8 \mathrm{~h}$ postinjection, the time of codeine/morphine administration (Figure $3 \mathrm{~b}$ ). Furthermore, there was no change in baseline nociception between prenicotine and $8 \mathrm{~h}$ postnicotine administration, consistent with the absence of nicotine or nicotine-induced nociception at this time point. Chronic nicotine administration also did not affect morphine analgesia (Figure 3e), indicating a lack of direct effect of nicotine on opioidinduced analgesia per se, including on the BBB permeability of morphine. Together, this substantially reduces the likelihood of nicotine pretreatment effects on general nociception, nicotine-mediated analgesia, or opioid-mediated analgesia in this chronic administration paradigm, suggesting instead an effect of nicotine on brain CYP2D levels.

Overall, these results suggest a role of brain CYP2D on codeine metabolism and analgesia. Following codeine administration, analgesia is initially mediated by morphine 
formed centrally by brain CYP2D, due to the delay in hepatically formed morphine entering the CNS. Thus lower (or higher) brain CYP2D activity and the consequent decrease (or increase) in brain morphine levels results in lesser (or greater) analgesia during this initial $30 \mathrm{~min}$ after codeine injection. Over time, morphine crosses into the brain, suggesting that analgesia at this later time period is mediated by morphine formed by hepatic CYP2D crossing into the CNS (seen from the shift in analgesic peak from morphine $(3.5 \mathrm{mg} / \mathrm{kg})$ compared with codeine $(20 \mathrm{mg} / \mathrm{kg}))$. The clinical implications of our findings are that individuals with higher brain CYP2D activity, such as genetically ultrarapid CYP2D metabolizers or smokers, may experience a quicker and greater onset of analgesia (and potentially abuse liability) from codeine, whereas those with lower brain CYP2D activity, such as non-smokers or those taking CYP2D inhibitors, may experience a delayed and lower analgesia. Accordingly, our results suggest that a translational study assessing the time course and peak of codeine's analgesic effects in smokers vs non-smokers, or within subject prenicotine/postnicotine treatment, would be warranted. Furthermore, it is suggested that drug metabolism within the brain may cause interindividual differences in drug response, which are not reflected in plasma drug levels.

\section{FUNDING AND DISCLOSURE}

This work was supported by the Endowed Chair in Addiction for the Department of Psychiatry (to RFT), Canadian Institutes of Health Research (TMD 132557, MOP 97751, and MOP 136937), Centre for Addiction and Mental Health and the CAMH Foundation, the Canada Foundation for Innovation (grant numbers 20289 and 16014), and the Ontario Ministry of Research and Innovation. Dr Rachel F Tyndale has consulted for McNeil and Apotex. Douglas M McMillan has no conflicts of interest to declare.

\section{ACKNOWLEDGMENTS}

We would like to thank Dr Sharon Miksys and Dr Bin Zhao for their invaluable technical support and scientific guidance and Fariba Baghai Wadji for her expert assistance with animal procedures.

\section{REFERENCES}

Adler TK, Fujimoto JM, Way EL, Baker EM (1955). The metabolic fate of codeine in man. J Pharmacol Exp Ther 114: 251-262.

Benowitz NL (1999). Nicotine addiction. Primary Care 26: 611-631. Berg CJ, Wen H, Cummings JR, Ahluwalia JS, Druss BG (2013). Depression and substance abuse and dependency in relation to current smoking status and frequency of smoking among nondaily and daily smokers. Am J Addiction 22: 581-589.

Berrendero F, Kieffer BL, Maldonado R (2002). Attenuation of nicotine-induced antinociception, rewarding effects, and dependence in mu-opioid receptor knock-out mice. J Neurosci 22: 10935-10940.

Bouw MR, Gardmark M, Hammarlund-Udenaes M (2000). Pharmacokinetic-pharmacodynamic modelling of morphine transport across the blood-brain barrier as a cause of the antinociceptive effect delay in rats-a microdialysis study. Pharm Res 17: 1220-1227.
Carstens E, Anderson KA, Simons CT, Carstens MI, Jinks SL (2001). Analgesia induced by chronic nicotine infusion in rats: differences by gender and pain test. Psychopharmacology 157: $40-45$.

Chen ZR, Irvine RJ, Bochner F, Somogyi AA (1990). Morphine formation from codeine in rat brain: a possible mechanism of codeine analgesia. Life Sci 46: 1067-1074.

Chen ZR, Somogyi AA, Reynolds G, Bochner F (1991). Disposition and metabolism of codeine after single and chronic doses in one poor and seven extensive metabolisers. Br J Clin Pharmacol 31: 381-390.

D'Amour FE, Smith DL (1941). A method for determining loss of pain sensation. J Pharmacol Exp Ther 72: 74-79.

Damaj MI, Meyer EM, Martin BR (2000). The antinociceptive effects of alpha7 nicotinic agonists in an acute pain model. Neuropharmacology 39: 2785-2791.

Dewey WL, Harris LS (1971). Antinociceptive activity of the narcotic antagonists analogues and antagonistic activity of the narcotic analgesics in rodents. J Pharmacol Exp Ther 179: 652-659.

Dhatt RK, Gudehithlu KP, Wemlinger TA, Tejwani GA, Neff NH, Hadjiconstantinou M (1995). Preproenkephalin mRNA and methionine-enkephalin content are increased in mouse striatum after treatment with nicotine. J Neurochem 64: 1878-1883.

Ding X, Kaminsky LS (2003). Human extrahepatic cytochromes P450: function in xenobiotic metabolism and tissue-selective chemical toxicity in the respiratory and gastrointestinal tracts. Annu Rev Pharmacol Toxicol 43: 149-173.

Dutra LM, Glantz SA (2014). High international electronic cigarette use among never smoker adolescents. J Adolesc Health 55: 595-597.

Eddy NB, Leimbach D (1952). Synthetic analgesics II. dithienylbutenyl- and dithienylbutylamines. J Pharmacol Exp Ther 107: 385-393.

Fischer B, Rehm J, Patra J, Cruz MF (2006). Changes in illicit opioid use across Canada. CMAJ 175: 1385.

Gaedigk A, Simon SD, Pearce RE, Bradford LD, Kennedy MJ, Leeder JS (2008). The CYP2D6 activity score: translating genotype information into a qualitative measure of phenotype. Clin Pharmacol Ther 83: 234-242.

Hiroi T, Chow T, Imaoka S, Funae Y (2002). Catalytic specificity of CYP2D isoforms in rat and human. Drug Metab Dispos 30: 970-976.

Kathiramalainathan K, Kaplan HL, Romach MK, Busto UE, Li NY, Sawe J et al (2000). Inhibition of cytochrome P450 2D6 modifies codeine abuse liability. J Clin Psychopharmacol 20: 435-444.

Khokhar JY, Tyndale RF (2011). Drug metabolism within the brain changes drug response: selective manipulation of brain CYP2B alters propofol effects. Neuropsychopharmacology 36: 692-700.

Khokhar JY, Tyndale RF (2012). Rat brain CYP2B-enzymatic activation of chlorpyrifos to the oxon mediates cholinergic neurotoxicity. Toxicol Sci 126: 325-335.

Krishna DR, Klotz U (1994). Extrahepatic metabolism of drugs in humans. Clin Pharmacokinet 26: 144-160.

Le Bars D, Gozariu M, Cadden SW (2001). Animal models of nociception. Pharmacol Rev 53: 597-652.

Mann A, Miksys S, Lee A, Mash DC, Tyndale RF (2008). Induction of the drug metabolizing enzyme CYP2D in monkey brain by chronic nicotine treatment. Neuropharmacology 55: 1147-1155.

Mann A, Miksys SL, Gaedigk A, Kish SJ, Mash DC, Tyndale RF (2012). The neuroprotective enzyme CYP2D6 increases in the brain with age and is lower in Parkinson's disease patients. Neurobiol Aging 33: 2160-2171.

Michels R, Marzuk PM (1993). Progress in psychiatry (1). N Engl J Med 329: $552-560$.

Miksys S, Tyndale RF (2004). The unique regulation of brain cytochrome P450 2 (CYP2) family enzymes by drugs and genetics. Drug Metab Rev 36: 313-333.

Miller LG (1990). Cigarettes and drug therapy: pharmacokinetic and pharmacodynamic considerations. Clin Pharm 9: 125-135. 
Miller RT, Miksys S, Hoffmann E, Tyndale RF (2014). Ethanol selfadministration and nicotine treatment increase brain levels of CYP2D in African green monkeys. Br J Pharmacol 171: 3077-3088.

Narimatsu S, Arai T, Masubuchi Y, Horie T, Hosokawa M, Ueno K et al (2001). Inactivation of rat cytochrome P450 2D enzyme by a further metabolite of 4-hydroxypropranolol, the major and active metabolite of propranolol. Biol Pharmaceut Bull 24: 988-994.

Oldendorf WH, Hyman S, Braun L, Oldendorf SZ (1972). Bloodbrain barrier: penetration of morphine, codeine, heroin, and methadone after carotid injection. Science 178: 984-986.

Pert CB, Snyder SH (1973). Properties of opiate-receptor binding in rat brain. Proc Natl Acad Sci USA 70: 2243-2247.

Richter KP, Ahluwalia JS (2000). A case for addressing cigarette use in methadone and other opioid treatment programs. J Addict Dis 19: 35-52.

Rowland K, Yeo WW, Ellis SW, Chadwick IG, Haq I, Lennard MS et al (1994). Inhibition of CYP2D6 activity by treatment with propranolol and the role of 4-hydroxy propranolol. $\mathrm{Br} J$ Clin Pharmacol 38: 9-14.

Schmidt BL, Tambeli CH, Gear RW, Levine JD (2001). Nicotine withdrawal hyperalgesia and opioid-mediated analgesia depend on nicotine receptors in nucleus accumbens. Neuroscience 106: 129-136.

Sindrup SH, Arendt-Nielsen L, Brosen K, Bjerring P, Angelo HR, Eriksen B et al (1992). The effect of quinidine on the analgesic effect of codeine. Eur J Clin Pharmacol 42: 587-591.

Sindrup SH, Brosen K, Bjerring P, Arendt-Nielsen L, Larsen U, Angelo HR et al (1990). Codeine increases pain thresholds to copper vapor laser stimuli in extensive but not poor metabolizers of sparteine. Clin Pharmacol Ther 48: 686-693.

Siu EC, Wildenauer DB, Tyndale RF (2006). Nicotine selfadministration in mice is associated with rates of nicotine inactivation by CYP2A5. Psychopharmacology 184: 401-408.
Skurtveit S, Furu K, Selmer R, Handal M, Tverdal A (2010). Nicotine dependence predicts repeated use of prescribed opioids. Prospective population-based cohort study. Ann Epidemiol 20: 890-897.

Tyndale RF, Droll KP, Sellers EM (1997). Genetically deficient CYP2D6 metabolism provides protection against oral opiate dependence. Pharmacogenetics 7: 375-379.

Vihavainen T, Piltonen M, Tuominen RK, Korpi ER, Ahtee L (2008). Morphine-nicotine interaction in conditioned place preference in mice after chronic nicotine exposure. Eur $J$ Pharmacol 587: 169-174.

Warner M, Gustafsson JA (1994). Effect of ethanol on cytochrome P450 in the rat brain. Proc Natl Acad Sci USA 91: 1019-1023.

Wu D, Otton SV, Inaba T, Kalow W, Sellers EM (1997). Interactions of amphetamine analogs with human liver CYP2D6. Biochem Pharmacol 53: 1605-1612.

$\mathrm{Xu}$ BQ, Aasmundstad TA, Bjorneboe A, Christophersen AS, Morland J (1995). Ethylmorphine O-deethylation in isolated rat hepatocytes. Involvement of codeine O-demethylation enzyme systems. Biochem Pharmacol 49: 453-460.

Yue J, Miksys S, Hoffmann E, Tyndale RF (2008). Chronic nicotine treatment induces rat CYP2D in the brain but not in the liver: an investigation of induction and time course. J Psychiatry Neurosci 33: 54-63.

Zanger UM, Raimundo S, Eichelbaum M (2004). Cytochrome P450 2D6: overview and update on pharmacology, genetics, biochemistry. Naunyn Schmiedebergs Arch Pharmacol 369: 23-37.

Zhou K, Khokhar JY, Zhao B, Tyndale RF (2013). First demonstration that brain CYP2D-mediated opiate metabolic activation alters analgesia in vivo. Biochem Pharmacol 85: 1848-1855.

Supplementary Information accompanies the paper on the Neuropsychopharmacology website (http://www.nature.com/npp) 